\title{
Toward improved effectiveness of bladder cancer immunotherapy
}

\author{
"Following the approval of ipilumamab, pembrolizumab and \\ nivolumab, several new checkpoint blockers are being currently tested \\ in multiple clinical trials and are expected to be approved in the near \\ future..."
}

\begin{abstract}
Keywords: bladder cancer $\bullet$ checkpoint blockade $\bullet$ chemokines $\bullet$ CTL $\bullet$ cytokines
- dendritic cells $\bullet$ immunotherapy $\bullet$ interferons $\bullet$ microenvironment $\bullet$ natural killer $\bullet$ NK

- stroma • tumor
\end{abstract}

Urothelial cancer (bladder cancer; $\mathrm{BCa}$ ) accounts for over 70,000 new cases and 15,000 deaths annually in USA. Although it can be successfully treated in its early stages, it has extremely high recurrence rates and becomes increasingly resistant to treatment at later stages. Intravesical BCG treatment reduces the recurrence rates of $\mathrm{BCa}$ from $60-70 \%$ to $30-40 \%$ and it is considered a prototypical target of cancer immunotherapy [1-3]. However, low overall durability of BCG responses beyond 2-3 years and the significant risk of progression to muscle invasive disease in spite of initial responses resulted in attempts to improve time to recurrence through BCG maintenance strategies [4]. Attempts to improve rates and the durability of BCG response, concerns over local toxicity, the risks of systemic infection in patients and risks of occupational exposure to healthcare workers due to the use of live bacterium have led to alternative strategies including reduced dosing and combining the isolated mycobacterial cell wall with mitochondrial DNA complexes, which have yielded early promising results [5]. Other immunotherapeutic strategies, such as single agent- or combination therapies involving IFN- $\alpha$, had disappointing results so far [6].

Recent US FDA approval of several new immunotherapeutic factors as treatments for other cancers highlights the overall advances of cancer immunotherapy and provides new venues for the improvement of long-term outcomes of BCa. Approval of Sipuleucel$\mathrm{T}$ (Provenge; Dendreon) as the first cellular product for treatment of advanced prostate cancer (a BCa trial has been initiated but not completed; ClinicalTrials.gov Identifier: NCT01353222), ipilumamab (Yervoy; Bristol-Myers Squibb), pembrolizumab (Keytruyda, MK-3475, Merck) and nivolumab (Opdivo; Bristol-Myers Squibb), antibodies blocking the immunosuppressive effects of the CTLA4 and PD1 pathways (checkpoint blockers) and of pegylated IFN- $\alpha$ (Sylatron; Merck) [7-9], demonstrate the effectiveness of different forms of immunotherapies targeting different aspects of anticancer immunity in multiple forms of cancer.

Available preclinical data provide rationale for combinatorial application of different modalities of immunotherapy to simultaneously target multiple phases of anticancer immunity, which are all suppressed, or at least inadequately activated, in cancer-bearing individuals.

Immune responses are initiated by activated antigen-presenting cells, such as dendritic cells (DCs), which provide lymph node-residing naive and memory $\mathrm{T}$ cells with 'signal 1' and 'signal 2' (respectively antigen and costimulatory signals). The process of DC activation can be initiated by both 'endogenous' factors (inflammatory factors of human origin, cytokines and interferons, including IFN- $\alpha$ ) and by 'exogenous' signals, such as toll-like-receptor (TLR) ligands present on multiple pathogens, including BCG, but typically missing from slowly developing tumors. The same process of DC activation can also instruct DC to induce the desirable killer/type 1 - effector $\mathrm{T}$ cell functions criti-

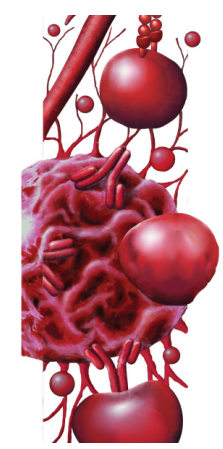

Pawel Kalinski

Departments of Surgery, Immunology, Bioengineering, Microbiology and Infectious Diseases, University of Pittsburgh, PA 15260, USA

and

University of Pittsburgh Cancer Institute, Hillman Cancer Center, UPCI Research Pavilion Suite 1.46, 5117 Center Ave, Pittsburgh, PA 152131863, USA

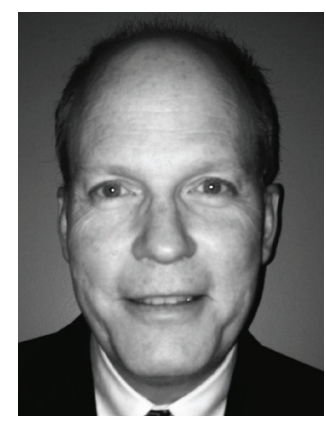

Jeffrey R Gingrich Author for correspondence: Department of Urology, University of Pittsburgh, PA 15260, USA and

University of Pittsburgh Cancer Institute, Cancer Pavilion Suite 209, 5200 Centre Ave., Pittsburgh, PA 15232, USA

Kalinskip@upmc.edu 
cal for effective cancer immunity ('signal 3' [10]), and to preferentially interact with specific immune cell subsets (Th1-, CD8 and NK cells rather than undesirable regulatory $\left.\mathrm{T}_{\mathrm{reg}}\right]$ cells [11]). Lastly, differentially activated DCs can induce different homing properties on activated $T$ cells $[12,13]$ ('signal 4'), allowing them to enter different tissues, including tumors, with different effectiveness. Since tumors are only weak activators of DCs and can even suppress DC activity, the spontaneous induction of anticancer immunity is usually ineffective.

\section{"An independent, but equally important factor critical for the antitumor effectiveness of immune cells is their ability to survive and function in the hostile tumor microenvironment."}

Following the induction of cancer-specific cells with the desirable (type-1) functions, the next factor critical for the effectiveness of antitumor immunity is the rate of entry of such effector cells into tumor tissues. Different types of immune cells are known to have different tumor-homing properties imprinted during their priming and reactivation [12]. While CTLs, Th1- and NK cells express high levels of CCR5 (the receptor for CCL3, CCL4 and CCL5) and CXCR3 (the receptor for CXCL9, CXCL10 and CXCL11), which allow them to effectively infiltrate infected tissues, the undesirable M2 macrophages, myeloid-derived suppressor cells (MDSCs) and $\mathrm{T}_{\text {regs }}$ express high levels of CCR2, CCR4, CXCR1 and CXCR4, which direct them to healing or chronically inflamed tissues, which produce high levels of their respective chemokine ligands: CCL2, CCL22, CXCL8 and CXCL12 [14,15]. Predictably, cancers produce mainly this second category of chemotactic factors, resulting in poor attraction of type-1 effector cells and their deactivation by the suppressor cells preferentially accumulating in tumor lesions, which limits the effectiveness of anticancer immunity [14,15].

An independent, but equally important factor critical for the antitumor effectiveness of immune cells is their ability to survive and function in the hostile tumor microenvironment. Most tumors (cancer cells, cancer-associated stromal cells and myeloid cells) produce high levels of homeostatic, tissue-protective, factors, typical of healing tissues. They include IL-10, TGF- $\beta$, VEGF, IL-6 IDO, arginase and COX2 and NOS2 products (such as prostaglandin $\mathrm{E}_{2} ; \mathrm{PGE}_{2}$, and nitric oxide; $\mathrm{NO}$ ), which all suppress the functions of spontaneously arising or therapy-induced immune cells and enhance the recruitment, expansion and activation of regulatory $\mathrm{T}$ cells $\left(\mathrm{T}_{\text {regs }}\right)$ and MDSCs [16]. Furthermore, the effectiveness of cancer immunity has been shown to be limited by 'checkpoint' control of CTL activity. Elevated levels of CTLA-4, PD-1, LAG-1 and TIM-3 on chronically activated T cells in cancer patients, and high levels of their ligands on cancer cells and tumor-associated antigen-presenting cells have been shown to control the expansion of cancerspecific cells, promote their apoptosis and suppression of effector functions [8,9].

Since the effectiveness of anticancer immune responses requires the involvement of all its components, long-term effectiveness of cancer immunotherapy is likely to benefit from simultaneous targeting of all the above tumor-associated suppressive mechanisms [17]. While the combinatorial approaches involving different classes of immunomodulating factors are still at early stage, their feasibility has been enhanced by the recent availability of multiple clinically approved factors active at each of the different steps of anticancer immunity.

Several strategies have been used to amplify the induction phase of anticancer immunity by systemically enhancing the DC activity in cancer patients. Among the known DC-activating cytokines, GMCSF and type-1 IFNs (particularly IFN- $\alpha$ ) have been widely tested as immune adjuvants to enhancing the effectiveness of immunization against cancerassociated antigens. Another potent DC-activating cytokine, TNF- $\alpha$, has been extensively studied for isolated limb perfusion in melanoma and sarcoma patients [18]. Among TLR ligands, different TLR3 ligands (dsRNA: poly-I:C and derivatives), TLR7/8 ligands (imiquimod, resiquimod) and TLR9 ligands (CpG oligonucleotides) are currently tested most intensively, with additional trials involving TLR2, TLR4 and TLR5 ligands) [19,20]. Ex vivo generation of mature DCs for subsequent adoptive transfer as 'DC vaccines' represents an alternative approach [7].

Incidentally, multiple factors used to activate DCs and to amplify the induction phase of type- 1 immunity (GM-CSF, IFN- $\alpha$, IFN- $\beta$, IFN- $\gamma$, TNF- $\alpha$, TLR ligands) can also modulate the production of chemokines in the tumor microenvironment (TME). However, two significant challenges related with their use in effective reprogramming of TME for effective anticancer immunity are their systemic, rather than tumor-selective effectiveness (ability of chemokines to function depends on the gradient of their concentrations and is lost in case of systemic chemokine induction); and limited selectivity of the currently used factors, which often just amplify the pre-existing undesirable pattern of chemokines within TME, rather than inducing the desirable chemokines [19-21]. In this situation, the efforts of multiple groups, including ours, have focused on developing new approaches to selectively induce effector-attracting chemokines 
in a tumor-selective fashion with concomitant local suppression of $\mathrm{T}_{\mathrm{reg}}$-attracting chemokines [21,22].

BCG has been shown to promote multiple levels of anti BCa immunity, but the documented association of its clinical effects with the levels of post-treatment leukocyturia [23] indicate the importance of local effects on TME and suggest that the factors enhancing the effector phase of anticancer immunity (enhancing tumor infiltration with immune cells and/or enhancing their local activity) can help improve the long-term outcomes. Since tumor infiltration with effector $\mathrm{CD} 8^{+}$ $\mathrm{T}$ cells (CTL) has been associated with good prognosis in $\mathrm{BCa}$ and other cancers [24,25], and intratumoral expression of chemokines is critical for effective CTL infiltration [15], we have recently evaluated the impact of BCG on local production of the CTL attractants versus the chemokines attracting undesirable MDSCs and $\mathrm{T}_{\text {reg }}$ cells [22]. Unexpectedly, we observed that BCG, used alone, not only failed to enhance local expression of CTL-attracting chemokines, CCL5 and CXCL10, but selectively enhanced MDSC- and $\mathrm{T}_{\text {reg }}$-attracting chemokines, CCL22 and CXCL8. These undesirable side-effects could be reversed by the combination of IFN- $\alpha$ and poly-I:C (TLR3 ligand), although not by the combination of BCG with IFN- $\alpha$ alone [22], raising the possibility that combinatorial factors including TLR3 ligands and IFN- $\alpha$ can enhance the effectiveness of the BCG-based and other forms of immunotherapy of $\mathrm{BCa}$ and, potentially, its combinations with checkpoint blockers. Importantly, the combination of BCG with IFN- $\alpha$ alone was ineffective in reprograming $\mathrm{BCa}$ microenvironment [22], helping to explain the disappointing clinical results of that combination [6], and suggesting the possibility that the inclusion of TLR3 ligands (or replacement of BCG with TLR3 ligands) may show improved efficacy.

Following the approval of ipilumamab, pembrolizumab and nivolumab, several new checkpoint blockers are being currently tested in multiple clinical trials and are expected to be approved in the near future [8,9], making checkpoint inhibition a particularly active field of cancer immunotherapy. In a recently completed Phase I study of a new PDL1 blocker (MPDL13280A) in 31 patients with metastatic $\mathrm{BCa}$, Powles et al. observed good tolerability of treatment and 54\% objective response rate in patients with PDL1-overexpressing $\mathrm{BCa}$ and $11 \%$ response rate in patients with PDL1-negative BCa [26]. Since the effectiveness of PD1/PDL1 blockade is known be predicted by the levels of local T-cell infiltration (IFN- $\gamma$-producing, type-1 $\mathrm{T}$ cells, enhance the PDL1 and PDL2 levels on cancer cells and local inflammatory cells), these data provide strong rationale for combining PD1/PDL1 blockade (and other checkpoint blockers) with factors which can enhance intratumoral levels of PDL1 [27], either directly or by increasing local attraction of IFN$\gamma$-producing immune cells into BCa lesions. Since the effectiveness of BCG can be enhanced by combination with IL-10 blockade [28], there is additional rationale for including additional countersuppressive factors (targeting IL-10, COX2/PGE 2 , VEGF, TGF- $\beta$, arginase, NOS2 or IDO) in the combination regimens. Although the relevance of the individual cancer-associated antigens to the immunogenicity of $\mathrm{BCa}$ is still unclear [29], prospective combinatorial approaches of BCa immunotherapy may also involve a vaccination component to synergistically target the induction- and effector-phases of anticancer immunity.

\section{Financial \& competing interests disclosure}

The authors acknowledge support from the $\mathrm{NIH}$ grant P01CA132714. The authors have no other relevant affiliations or financial involvement with any organization or entity with a financial interest in or financial conflict with the subject matter or materials discussed in the manuscript apart from those disclosed.

No writing assistance was utilized in the production of this manuscript.

\section{References}

1 Herr HW, Morales A. History of bacillus Calmette-Guerin and bladder cancer: an immunotherapy success story. J. Urol. 179(1), 53-56 (2008).

2 Gofrit ON, Pode D, Pizov G et al. The natural history of bladder carcinoma in situ after initial response to bacillus Calmette-Guerin immunotherapy. Urol. Oncol. 27(3), 258-262 (2009).

3 Zhu S, Tang Y, Li K et al. Optimal schedule of bacillus Calmette-Guerin for non-muscle-invasive bladder cancer: a meta-analysis of comparative studies. BMC Cancer 13, 332 (2013).

Kamat AM, Flaig TW, Grossman HB et al. Expert consensus document: consensus statement on best practice management regarding the use of intravesical immunotherapy with BCG for bladder cancer. Nat. Rev. Urol. 12(4), 225-235 (2015).

5 Morales A, Herr H, Steinberg G et al. Efficacy and safety of MCNA in patients with nonmuscle invasive bladder cancer at high risk for recurrence and progression after failed treatment with bacillus Calmette-Guerin. J. Urol. 193(4), 1135-1143 (2015).

6 O’Donnell MA, Lilli K, Leopold C. National Bacillus Calmette-Guerin/Interferon Phase 2 Investigator Group. Interim results from a national multicenter Phase II trial of combination bacillus Calmette-Guerin plus interferon alfa- 
2b for superficial bladder cancer. J. Urol. 172(3), 888-893 (2004).

7 Kirkwood JM, Butterfield LH, Tarhini AA, Zarour H, Kalinski P, Ferrone S. Immunotherapy of cancer in 2012. CA Cancer J. Clin. 62(5), 309-335 (2012).

8 Topalian SL, Drake CG, Pardoll DM. Immune checkpoint blockade: a common denominator approach to cancer therapy. Cancer Cell 27(4), 450-461 (2015).

9 Sharma P, Allison JP. The future of immune checkpoint therapy. Science 348(6230), 56-61 (2015).

10 Kalinski P, Hilkens CM, Wierenga EA, Kapsenberg ML. T-cell priming by type- 1 and type- 2 polarized dendritic cells: the concept of a third signal. Immunol. Today 20(12), 561-567 (1999).

11 Muthuswamy R, Urban J, Lee JJ, Reinhart TA, Bartlett D, Kalinski P. Ability of mature dendritic cells to interact with regulatory $\mathrm{T}$ cells is imprinted during maturation. Cancer Res. 68(14), 5972-5978 (2008).

12 Weninger W, Manjunath N, von Andrian UH. Migration and differentiation of $\mathrm{CD}^{+} \mathrm{T}$ cells. Immunol. Rev 186, 221-233 (2002).

13 Watchmaker PB, Berk E, Muthuswamy R et al. Independent regulation of chemokine responsiveness and cytolytic function versus $\mathrm{CD} 8^{+} \mathrm{T}$ cell expansion by dendritic cells. J. Immunol. 184(2), 591-597 (2010).

14 Mantovani A, Allavena P, Sozzani S, Vecchi A, Locati M, Sica A. Chemokines in the recruitment and shaping of the leukocyte infiltrate of tumors. Semin. Cancer Biol. 14(3), 155-160 (2004).

15 Gajewski TF, Schreiber H, Fu YX. Innate and adaptive immune cells in the tumor microenvironment. Nature Immunol. 14(10), 1014-1022 (2013).

16 Rabinovich GA, Gabrilovich D, Sotomayor EM. Immunosuppressive strategies that are mediated by tumor cells. Annu. Rev. Immunol. 25, 267-296 (2007).

17 Spranger S, Gajewski T. Rational combinations of immunotherapeutics that target discrete pathways. J. Immunother. Cancer 1, 16 (2013).

18 Van Horssen R, Ten Hagen TL, Eggermont AM. TNF-alpha in cancer treatment: molecular insights, antitumor effects, and clinical utility. Oncologist 11(4), 397-408 (2006).
19 Kaczanowska S, Joseph AM, Davila E. TLR agonists: our best frenemy in cancer immunotherapy. J. Leukoc. Biol. 93(6), 847-863 (2013).

20 Lu H. TLR Agonists for Cancer Immunotherapy: tipping the balance between the immune stimulatory and inhibitory effects. Front. Immunol. 5, 83 (2014).

21 Muthuswamy R, Berk E, Junecko BF et al. NF-kappaB hyperactivation in tumor tissues allows tumor-selective reprogramming of the chemokine microenvironment to enhance the recruitment of cytolytic T effector cells. Cancer Res. 72(15), 3735-3743 (2012).

22 Muthuswamy R, Wang L, Pitteroff J, Gingrich JR, Kalinski P. Combination of IFNalpha and poly-I:C reprograms bladder cancer microenvironment for enhanced CTL attraction. J. Immunother. Cancer 3, 6 (2015).

23 Saint F, Patard JJ, Irani J et al. Leukocyturia as a predictor of tolerance and efficacy of intravesical BCG maintenance therapy for superficial bladder cancer. Urology 57(4), 617-621; discussion 621-612 (2001).

24 Sharma P, Shen Y, Wen S et al. CD8 tumor-infiltrating lymphocytes are predictive of survival in muscle-invasive urothelial carcinoma. Proc. Natl Acad. Sci. USA 104(10), 3967-3972 (2007).

25 Fridman WH, Pages F, Sautes-Fridman C, Galon J. The immune contexture in human tumours: impact on clinical outcome. Nat. Rev. Cancer 12(4), 298-306 (2012).

26 Powles T, Eder JP, Fine GD et al. MPDL3280A (anti-PD-L1) treatment leads to clinical activity in metastatic bladder cancer. Nature 515(7528), 558-562 (2014).

27 Powles T. Immunotherapy: the development of immunotherapy in urothelial bladder cancer. Nat. Rev. Clin. Oncol. 12(4), 193-194 (2015).

28 Newton MR, Askeland EJ, Andresen ED et al. Antiinterleukin-10R1 monoclonal antibody in combination with bacillus Calmette-Guerin is protective against bladder cancer metastasis in a murine orthotopic tumour model and demonstrates systemic specific anti-tumour immunity. Clin. Exp. Immunol. 177(1), 261-268 (2014).

29 Komohara Y, Harada M, Arima Y et al. Anti-cancer vaccine candidates in specific immunotherapy for bladder carcinoma. Int. J. Oncol. 29(6), 1555-1560 (2006). 